\title{
Chromosome Studies in 150 Sexually Abnormal Patients (A Summarized Report) ${ }^{1,2}$ Part I
}

\author{
Sajiro Makino
}

Zoological Institute, Hokkaido University, Sapporo, Japan

\author{
Received October 12, 1965
}

During the past several years, following the findings of chromosomal abnormality in Down's syndrome patients by Lejeune et al. (1959), as well as those by Ford et al. (1959) and by Jacobs and Strong (1959) in sexually abnormal patients, and promoted primarily by recent technical advances in cytogenetics, a number of investigators have expanded enthusiastic effort in screening congenital or hereditary disorders and clinical defects for chromosome abnormalities in human beings. To date, current authors have furnished critical information which provides a series of significant associations between chromosomal aberrations and disease states.

Since 1959 a survey on chromosomal abnormality in various congenital or hereditary syndromes and in clinical disorders in Japanese people has been conducted by the author in cooperation with his collaborators as well as clinicians working in many medical fields. The author (Makino 1964) summarized data accumulated during a period from 1959 to 1963 on 300 subjects with congenital diseases, clinical defects and sexual anomalies, together with discussion on reported subjects by current authors. Considerable amount of chromosomal data has been collected since then on sexually abnormal human subjects. The subjects of these studies have been already described, or are soon to be published in some 20 separate papers. In the present article the author wishes to summarize those described cases in terms of cytogenetic aspects, for the sake of convenience for reference, with the principal aim of providing basic information available for understanding the relationship of clinical conditions to cytogenetic features, as well as to serve as a future clue for better criteria for differential diagnosis of abnormal sexual development in man.

This record covers information on the chromosomes obtained in 150 cases of sexually abnormal individuals during a period from September 1959 to the end of July 1965 in the author's laboratory. The majority of cases have already been published or are to be published by the author and/or his colleagues in conjunction with clinicians in pediatrics, urology, obstetrics and gynecology, and some others. Some data which has already been summarized by the author (Makino 1964) are included in this article together with recent data, since they are essential as supplementary material for clinical consideration. It should be mentioned, however, that rapidly expanding present-day human cytogenetics has rendered it difficult to make any generalization underlying etiological problems partaining to sexual anomalies in man.

In order to arrange data relevant to the description of chromosome abnormalities and their correlation with phenotypic effects in the present subjects, as well as in the future material, the cases under study were tentatively classified in several groups in relation to disease states and chromosomal conditions as follows:--

\section{Phenotypic males}

Klinefelter's syndrome with an XXXXY sex chromosome complement (Case \#1).

1 Contribution No. 770 from the Zoological Institute, Faculty of Science, Hokkaido University, Sapporo, Japan.

2 With the cooperation of Dr. Motomichi Sasaki in organizing data. 
Klinefelter's syndrome with an XXXY sex chromosome complement (Case \#2).

Klinefelter's syndrome with an XXYY sex chromosome complement (Case \#3).

Klinefelter's syndrome with an XX/XXY sex chromosome complement (Case \#4).

Klinefelter's syndrome with an XXY sex chromosome complement (Cases \#5-\#33).

Suspected Klinefelter's syndrome with an XY sex chromosome complement (Cases \#34-\#36).

Patients with hypospadias (Cases $\# 37-\# 45$ ).

Patients with cryptorchism (Cases $\# 46-\# 70$ ).

Patients with gynecomastia (Cases \#71-\#74).

Patients with an $\mathrm{XO} / \mathrm{XY}$ sex chromosome complement (Cases \#75-\#77).

Miscellaneous sex abnormalities (Cases $\# 78-\# 96$ ).

Patients with azoospermia or oligospermia (Cases \#97-\#113).

Phenotypic females

Turner's syndrome (Cases \#114-\#116).

Patients with an enlarged clitoris (Cases \#117-\#129).

Patients without vagia (Cases \#130-\#136).

Adrenogenital syndrome (Cases \#137-\#142).

Miscellaneous sex abnormalities (Cases \#143-\#145).

Testicular feminization (Cases \#146-\#148).

True hermaphroditism and agonadism

True hermaphroditism (Case $\# 149$ ).

Agonadism (Case \#150).

\section{GENERAL REMARKS}

\section{Phenotypic males}

Klinefelter's syndrome. Patients generally designated by the term Klinefelter's syndrome are infertile males characterized by azoospermia, gynecomastia, elevated urinary gonadotropin excretion and atrophic testes together with degeneration and hyalinization of the seminiferous tubules and hyperplasia of Leydig cells, and with a female-type sex chromatin pattern. In general, patients possess a chromosome number of 47 and an XXY sexchromosome complement. However, this is by no means the only abnormal sex chromosome constitution for this syndrome. So far as the author is aware, some 15 variants of sex-chromosome complexes other than the regular XXY-type have been reported to occur in chromatin positive Klinefelter's cases (Makino et al. 1964b). In the present survey, 33 cases of this syndrome were subjected to chromosomal study. Among the subjects under study there was 1 case with an XXXXY sex chromosome complement, 1 with an XXXY, 1 with an XXYY, 29 with an XXY, and 1 with an XX/ $\mathrm{XXY}$ mosaicism.

There is another group of patients which is referred to as the so-called chromatin-negative Klinefelter's syndrome. The clinical feature of these patients are similar to those of chromatin-positive cases. Three cases (Cases \#34-\#36) dealt with in the present report under the designation of suspected Klinefelter's syndrome are probably of this category. Chromosome analyses revealed that they had a normal male complement as $46-\mathrm{XY}$. 
Referring to clinical data presented in this report and in some others, it is apparent that any one of the clinical symptoms such as azoospermia, gynecomastia, eunuchoid body proportions and atrophic testes, is not necessarily specific to a given genotypical constitution of this syndrome. Court Brown et al. (1964) adopted a term " seminiferous tubule dysgenesis" as the most satisfactory term to indicate chromatin-positive cases of Klinefelter's syndrome. In the present report, however, the classical term was adopted, holding the sex-chromosome complement as the best designation.

Patients with hypospadias. Nine patients predominantly affected by hypospadias in different degrees were chromosomally screened. Eight of the 9 patients were revealed to have an apparently normal male karyotype, 46$X Y$. The remaining one patient (Case $\$ 39$ ) was found to have an unusually long $\mathrm{Y}$ chromosome, other elements being normal in appearance. The length of the abnormal $\mathrm{Y}$ chromosome corresponded approximately to that of group 16-18 chromosomes. However, the mention should be made here that a long $\mathrm{Y}$ chromosome is not always connected with a specific phenotypic abnormality (Makino et al. 1963c, Makino and Muramoto 1964, Makino and Takagi, 1966).

Patients with cryptorchism. Twenty-five patients characterized by bilateral or unilateral undescended testes were subjected to chromosome studies. In the last two cases (Cases $\$ 69$ and $\# 70$ ), however, the testis was found on the left side only, the right testis being absent. All the patients except for case \#46 were found to have a normal male karyotype, 46-XY. The exceptional case was remarkable by having an unusually enlarged short arm in one of the no. 15 chromosomes, other elements being normal as a male karyotype (Kato et al. 1965). The same abnormality was found in his father, paternal grandfather, a female cousin and two paternal aunts, all of them being phenotypically normal and healthy (Makino et al. 1966). Although the origin and nature of the aberrant chromosome remains unknown at the present status of knowledge, it is very apparent that it is heritable and is not always associated with a specific phenotypical abnormality, like the feature occurring in the increased length of the $\mathrm{Y}$ chromosome.

Patients with gynecomastia. Four patients who suffered mostly from gynecomastia were included in this category. They all had a normal male complement, 46-XY.

Patients with an $X O / X Y$ sex chromosome complement. Since a mosaic constitution, $\mathrm{XO} / \mathrm{XY}$, seems to exert variable effects on the sexual phenotype, depending on the difference in relative proportions of the two cell lines from subject to subject, the patients do not always exhibit a common phenotypic similarity. One general feature in all cases of an XO/XY mosaicism so far reported is the presence in some form of gonadal dysgenesis (Robinson et al. 1964). However, one case (Case \#75) out of the three dealt with in the present report differs from the other cases in having functioning testes. 
Further discussion on this problem has been given in another paper (Dave, 1966).

Miscellaneous sexual abnormalities. Under this heading, were described chromosome conditions of 19 patients with anomalous external genitalia, variable in manifestation and degree, and with or without other features such as eunuchoidism, gynecomastia, abnormal hair distribution and mental retardation. All the cases showed a normal male karyotype, 46-XY, with one exceptional case in which an unusually long $\mathrm{Y}$ chromosome was detected, while other elements were normal in appearance. The length of the $Y$ corresponded to that of group 16-18 chromosomes. The patient's father, who was normal and healthy, also had a long $\mathrm{Y}$ of apparently similar nature.

Patients with azoospermia or oligospermia. Seventeen patients who complained of infertility due to azoospermia or oligospermia were collected here. They are apparently normal-looking men with normal or almost normal external genitalia. A normal male karyotype was established in 16 out of the 17 cases. The remaining one case (Case $\$ 101$ ) was characterized by a long $\mathrm{Y}$ chromosome corresponding in length to group 19-20 chromosomes. The other elements, however, were normal in number and morphology.

\section{Phenotypic females}

Turner's syndrome. Phenotypic females generally referred to as Turner's syndrome are characterized by ovarian dysgenesis with small stature and multiple congenital abnormalities, such as webbing of the skin of the neck and bilateral cubitus valgus. They are usually chromatin-negative and noted by having an XO sex-determining mechanism. In the present report, three patients were subjected to chromosomal diagnosis. The first case (Case \#114) showed no neck webbing, while it occurred in the other two (Cases \#115 and \#116). All the three patients showed a negative sex-chromatin pattern. Chromosome analyses revealed that all of them had 45 chromosomes with a regular XO sex-mechanism.

Patiens with an enlarged clitoris. Most of the patients here studied were babies or prepuberal girls who were hospitalized due to an enlarged clitoris. Thirteen patients subjected to chromosomal examination were found to possess a normal female complement, i.e., 46 chromosomes with an $\mathrm{XX}$ sexdetermining mechanism.

Patients without vagina. Seven female patients, whose chief complaint was primary amenorrhea and absence of the vagina, were chromosomally examined and were shown to possess a normal female karyotype, 46-XX. Jacobs et al. (1961) have found a remarkably high incidence of sex-chromosome abnormalities in a group of women with primary amenorrhea. Referring to the data presented in their study and in the present survey, however, it seems probable that a simple symptom of vaginal defects is not always associated with sex-chromosome aberrations. 
Adrenogenital syndrome. This syndrome is a condition of pseudohermaphroditism or virilism induced endogenously by increased secretion of

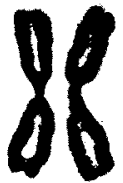

I

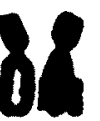

6

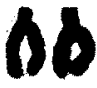

13

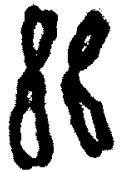

2

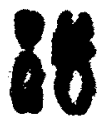

7

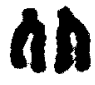

14

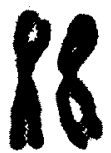

3

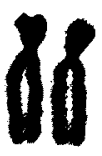

4

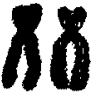

10

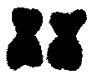

16

\section{$88 \quad 28$}

19 20

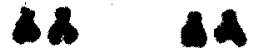

21 22

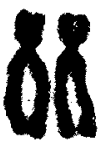

5

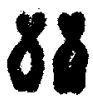

12
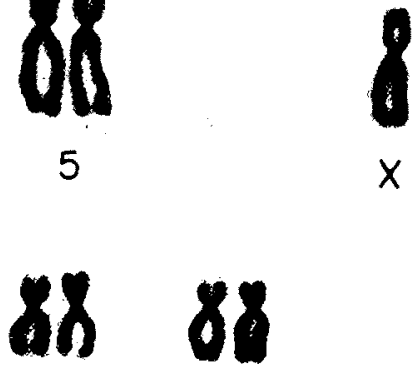

11

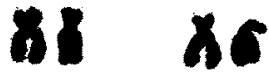

17

Fig. 1. Normal male karyotyfe, 46-XY, in a patient with hypospadias (Case \#42).

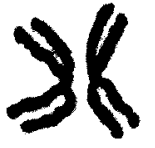

I

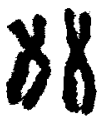

6

on

13

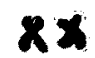

19

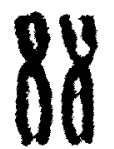

2

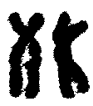

7

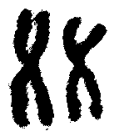

3

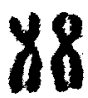

8

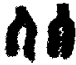

15

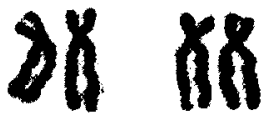

4
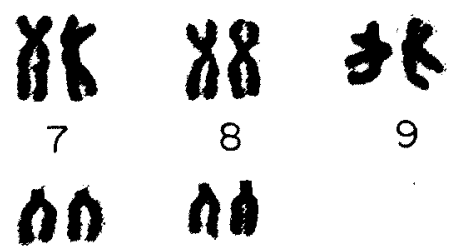

14

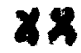

20
9

$x y$

10

34

16

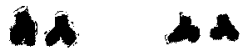

21
14

$x \times$

Fig. 2. Normal female karyotype, $46-\mathrm{XX}$, in a patient with an enlarged clitoris (Case $\# 127$ ). androgens from the adrenal gland of the patient. Depending on the sex of the patient and the time at which the pathological secretion of androgens begins a variety of clinical pictures is produced (Bierich 1963). Six female 
patients with this syndrome were chromosomally investigated and all were found to consistently possess 46 chromosomes with an XX sex-determining mechanism, i.e., a normal female karyotype.

Miscellaneous sex abnormalities. Three cases each with sexual hypoplasia, hypoplastic uterus and epispadia, were screened here for the chro-

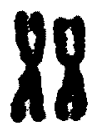

।

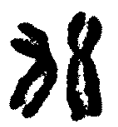

2

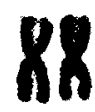

3

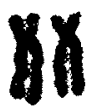

4

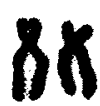

5 $x+x y$

$x \times x \times$

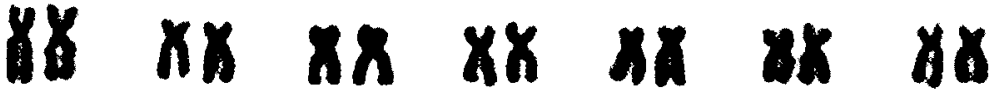

$\begin{array}{lllllll}6 & 7 & 8 & 9 & 10 & 11 & 12\end{array}$
180

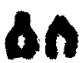
ax Ak
13
14
15
16
17
18 \\ A}

138

14

19

20

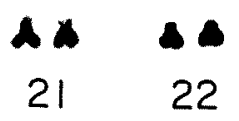

Fig. 3. A 49-XXXXY karyotype in a patient with Klinefelter's syndrome (Case \#1).

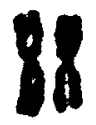

I

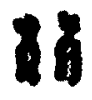

6

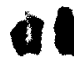

13

ax

19

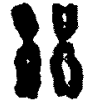

2

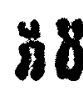

7

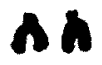

14

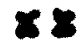

20

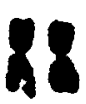

3

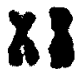

8

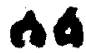

15

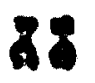

9

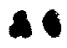

21

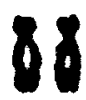

4

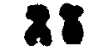

16

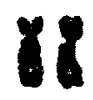

5

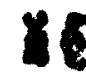

10

38

80

17
888

$x \times x$

Fig. 4. A 48-XXXY karyotype in a patient with Klinefelter's syndrome (Case \#2).

mosomal abnormality. The first one (Case \#143), who was diagnosed as sexual hypoplasia, was remarkable by having 47 chromosomes with an XXX sex-chromosome constitution. The other two cases were chromosomally normal having 44 apparently normal autosomes and a pair of sex chromosomes, XX. 
Testicular feminization. Testicular feminization is a form of clearly demarkated intersex that is characterized by completely female external genitalia, well-developed breast but without a uterus and with underdeveloped or maldeveloped testes (Hauser 1963). Recent cytogenetical studies have made

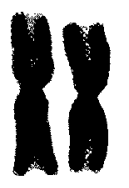

I
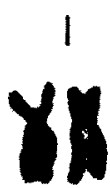

6

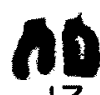

13

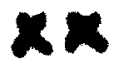

19

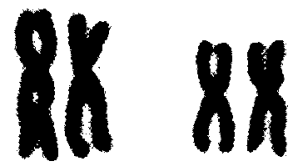

3

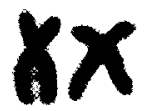

7

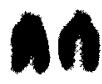

14

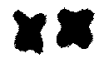

20

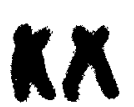

8

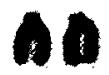

15

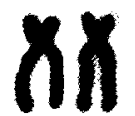

4

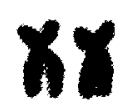

9

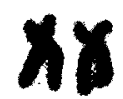

10

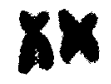

16

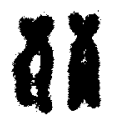

5

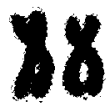

$x x$

Fig. 5. A 48-XXYY karyotype in a patient with Klinefelter's syndrome (Case \#3).

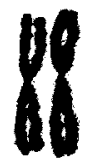

I

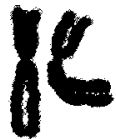

2

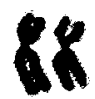

7

6
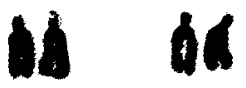

14

13

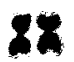

19

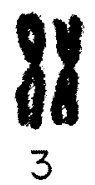

88

8

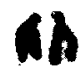

15

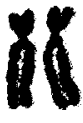

4

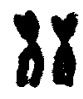

9

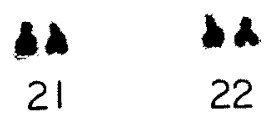

10

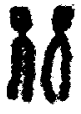

5
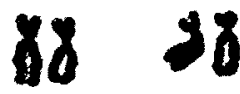

II

12

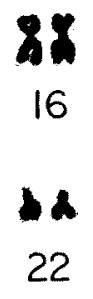

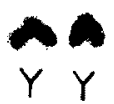

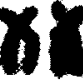

12

18

Fig. 6. A 47-XXY karyotype in a partient with Klinefelter's syndrome (Case $\$ 4$ ).

it clear that they have a negative sex-chromatin and a normal male chromosome complement. Three intersex patients clinically diagnosed as testicular feminization were chromosomally examined here: they were found to have an apparently normal male karyotype, $46-\mathrm{XY}$. 
True hermaphroditism and agonadism

True hermaphroditism. True hermaphroditism is a condition of abnormal sex development characterized by intersexual phenotype, carrying both ovarian
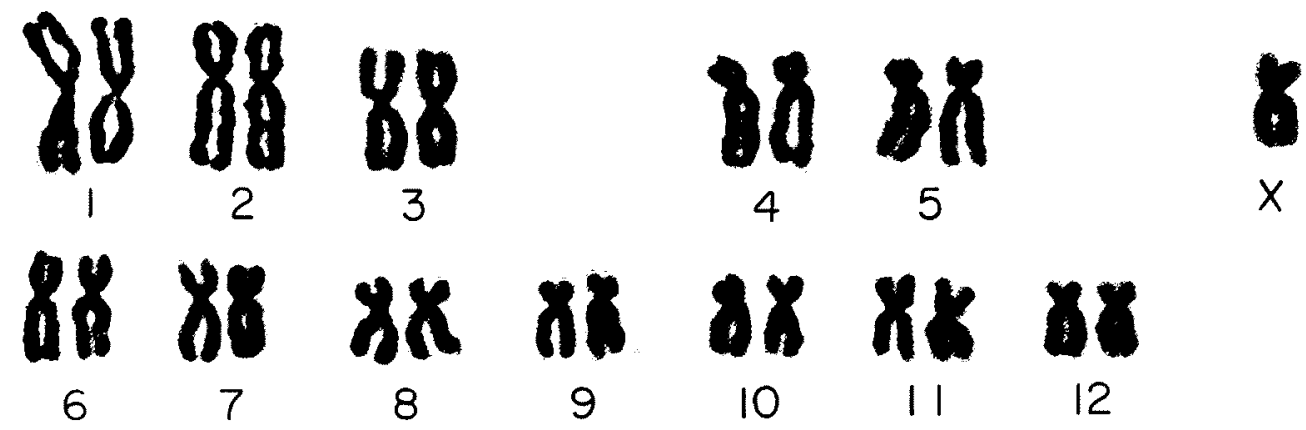

8

9
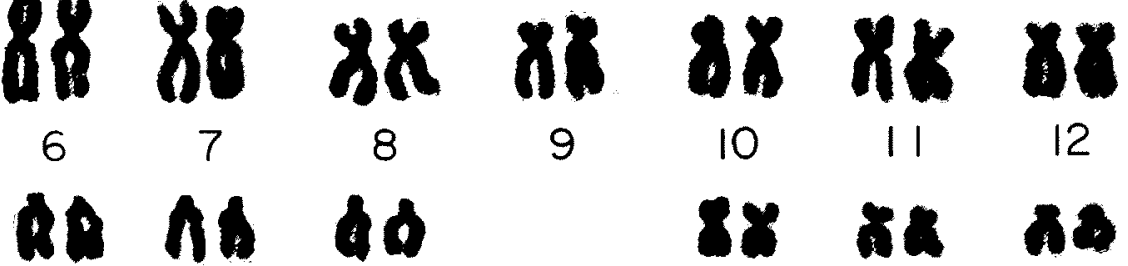

15

10

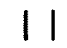

12

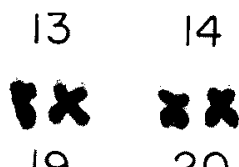

1920
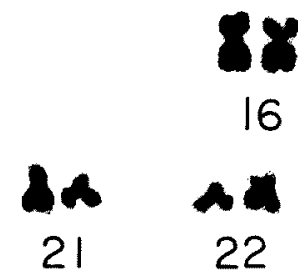

Fig. 7. A 45-XO karyotype in a patient with Turner's syndrome (Case \#116).
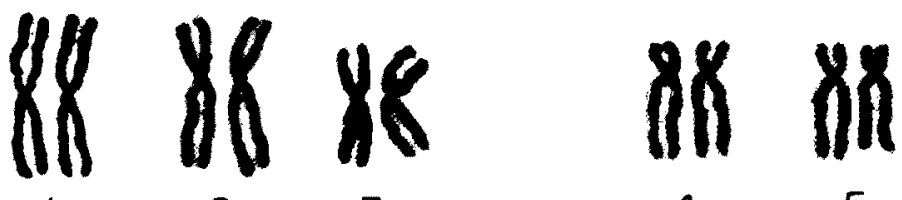

'Mi

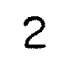

3

4

5

$x \times x$
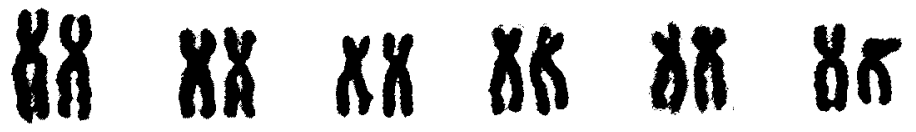

68

6 7

8

9

10

11

12

o6

13 14

15

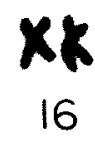

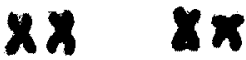

An

19

20

21

$x \mathrm{n}$

17

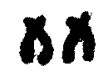

18

Fig. 8. A $47-\mathrm{XXX}$ karyotype in a patient with sexual hypoplasia (Case $\# 143$ ).

and testicular tissues diagnosed histologically. Based on squashed preparations from bone marrow biopsies, a case of true hermaphroditism chromosomally studied here revealed the occurrence of an apparently normal female complex, 46-XX. Recently, Hungerford et al. (1964) re-investigated, with both blood 
and skin cultures, the chromosomes of a subject diagnosed as true hermaphroditism, in which they found in 1959 a 46 -chromosome, XX pattern in blood cultures, and confirmed the former finding of a non-mosaic condition.

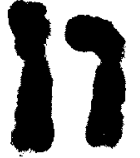

I

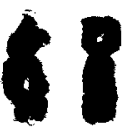

6

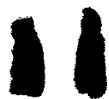

13

88

19

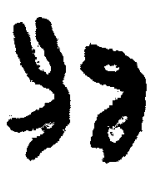

1

14

6

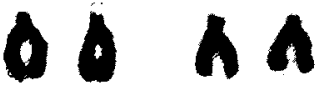

13

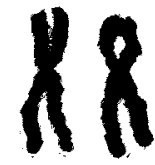

2

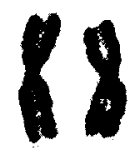

3

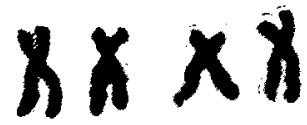

8

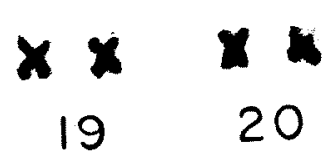

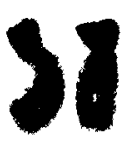

4

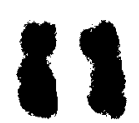

8

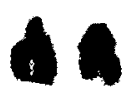

15

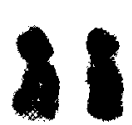

9

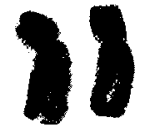

5

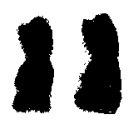

$x \times$

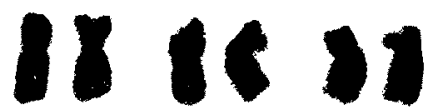

12

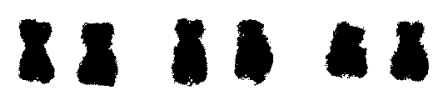

$\begin{array}{lll}16 & 17 \quad 18\end{array}$
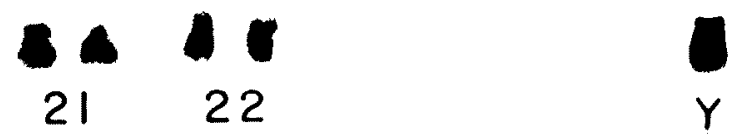

Y
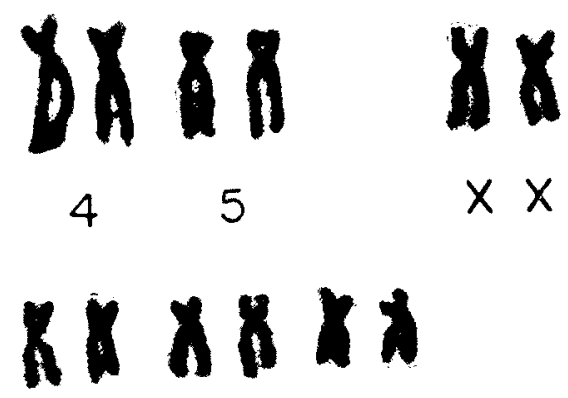

$10 \quad 11 \quad 12$
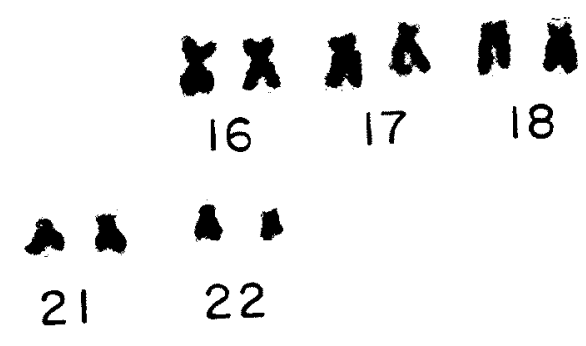

Fig. 9. A 47-XXY/46-XY karyotype in a patient with Klinefelter's syndrome (Case \#4). Top, 47-XXY cell. Bottom, 46-XX cell.

Agonadism. A 3-year-old patient who had no internal sexual organs was subjected to chromosomal diagnosis. It was shown that this patient had a normal male karyotype, 46-XY. 


\section{Conclusion and summary}

With the epoch-making work by Tjio and Levan on human chromosomes in 1956, a new era began in human cytogenetics, and from then on considerable effort has been devoted to define the morphology of the normal

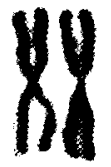

I

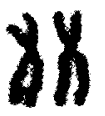

6

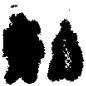

13

21

19

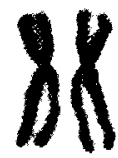

2

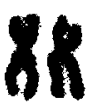

7

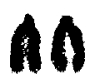

14

ix

20
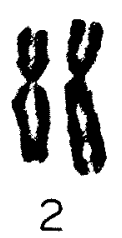

88

6

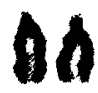

13

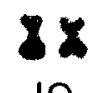

19

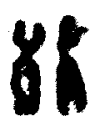

7

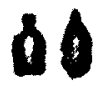

14

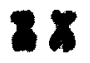

20

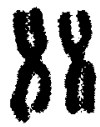

3

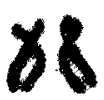

8

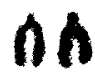

15

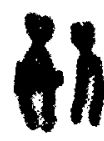

4

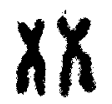

9
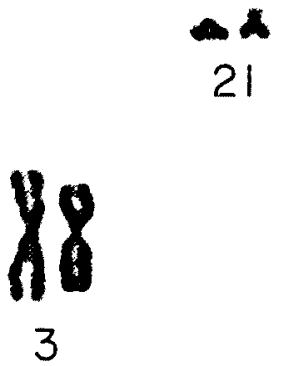

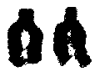

15

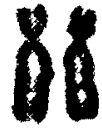

4

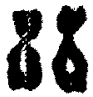

9

8
11

10

\& $6 x$

16

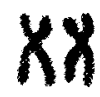

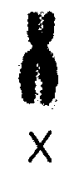

5

7)

12

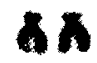

18

Fig. 10. A 45-XO/46-XY karyotype in a sexually abnormal male (Case $\# 77$ ). Top, 46-XY cell. Bottom, 45-XO cell.

karyotype in various human races by a number of investigators, as well as to detect association between chromosomal aberrations and congenital disorders and/or clinical defects. These studies still continue and yield a good deal of information available for differential diagnosis, or for critical understanding of the etiological cause of those diseases. In recent years, human clinical 
genetics stems to a large degree from remarkable contributions to basic genetic knowledge by research in these fields. To date, chromosomal analysis of somatic cells is essential in clinical fields in man. Particularly, sex chromatin tests and direct examination of sex chromosomes are now indispensable tools available for diagnosis of the genetic sex, and contribute to an approach toward etiological analysis of the cause or rise of abnormal sexual development.

Prompted by the work of Ford et al. (1959) and of Jacobs and Strong (1959), evidence was presented to show that various types of sexual abnormalities or defects of sexual development in man were associated in most cases with sex chromosome abnormalities. Ever-increased knowledge of human

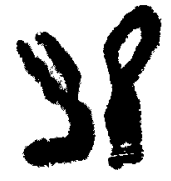

1

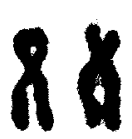

7

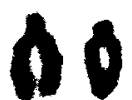

13

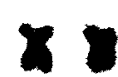

19

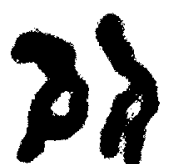

2

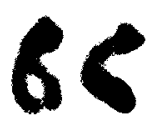

8

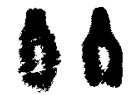

14

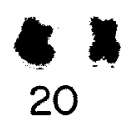

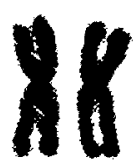

3

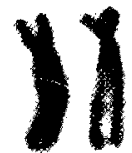

4

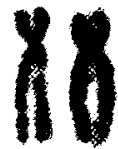

5

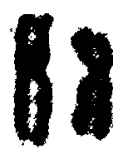

6
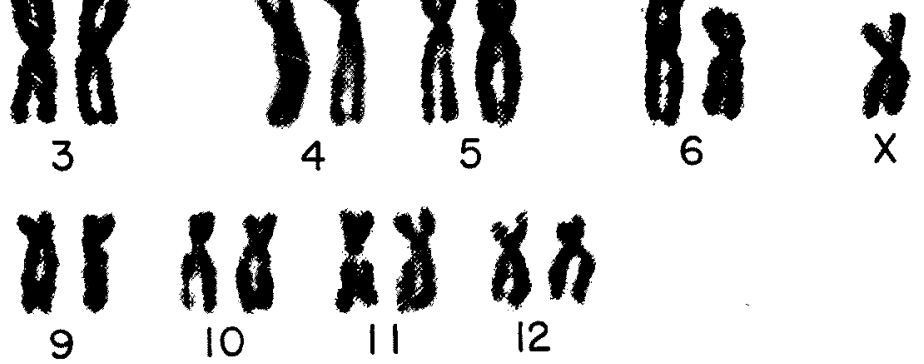

18

12

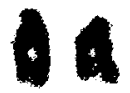

15

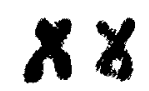

16

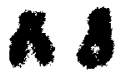

17

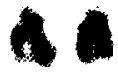

18
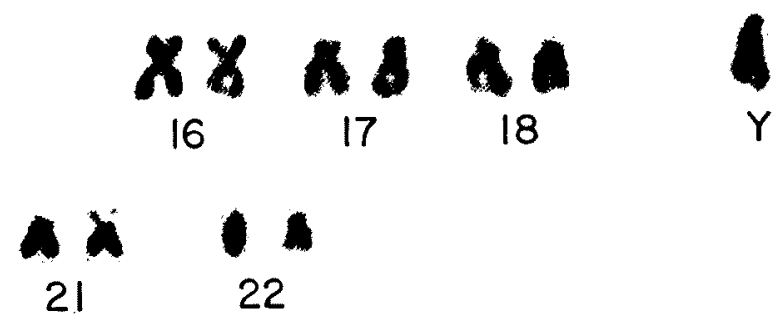

Fig. 11. Karyotype analysis showing an unusually long $\mathrm{Y}$ chromosome in a sexually abnormal male (Case $\# 96$ ).

cytogenetics has been catalogued in many recent reviews, particularly in medical interests. Those involving problems exclusively of sexual abnormalities are reviews by Harnden and Jacobs (1961), Hamerton (1961), Miller (1964), Court-Brown et al. (1964), Armstrong (1964), and others.

Intersex in man has long been a subject of special attention since the Greek and Roman Ages and a great deal of phenotypic variants in this condition have been described. Available data have well established that, though the XX-XY sex-determining mechanism is recognized as a basic principle of normal sex determination, there exists a large number of numerical and structural varieties of sex-chromosome aberrations in association with human sexual abnormalities. Abnormal numbers of sex chromosomes usually occur as simple aneuploidy, with a single missing or gaining of one or more sex elements. Representatives of such abnormal sex-chromosome constitutions so far reported are represented by XO, XXX, XXXX, XXXXX, 
$\mathrm{XXY}, \mathrm{XXXY}, \mathrm{XXXXY}, \mathrm{XYY}, \mathrm{XXYY}, \mathrm{XXXYY}$, and so on. As examples of reported structural abnormalities are deleted $\mathrm{X}$ or $\mathrm{Y}$ chromosomes, $\mathrm{X}$ or $\mathrm{Y}$ isochromosomes, ring $\mathrm{X}$ chromosomes and unusually long or short $\mathrm{Y}$ chromosomes occurring in sexually abnormal patients, as well as in phenotypically normal human beings. In addition to the above abnormalities, sex: chromosome mosaicism has been known to be essential in the cause or rise of some abnormal sexual development (Källén and Levan 1962, Dave 1966). Though less frequently, sex-chromosome mosaics such as $\mathrm{XX} / \mathrm{XXY}, \mathrm{XY} / \mathrm{XXY}$, $\mathrm{XY} / \mathrm{XXXY}, \mathrm{XXXY} / \mathrm{XXXXY}, \mathrm{XXXY} / \mathrm{XXXXY} / \mathrm{XXXXXY}, \mathrm{XXY} / \mathrm{XX} / \mathrm{XY} / \mathrm{XO}$, $\mathrm{XXY} / \mathrm{XXXY}, \mathrm{XO} / \mathrm{XX}, \mathrm{XO} / \mathrm{XY}, \mathrm{XO} / \mathrm{XXX}, \mathrm{XO} / \mathrm{XYY}, \mathrm{XO} / \mathrm{XX} / \mathrm{XXX}, \mathrm{XO} / \mathrm{XY}$ /XX, XO/XY/XYY, XO/XXY, XO/Xy, XX/XY, XX/XXX, XX/XXX/XXXX, $\mathrm{XX} / \mathrm{XXY} / \mathrm{XXYYY}, \mathrm{XXXX} / \mathrm{XXXXY}$, and so on, have been described in recent literature. The clinical picture in a series of mosaics is usually more variable than in a series of individuals with simple monosomy or trisomy, etc., because each cell line may tend to produce a different and a phenotypically distinct group of aberrations (Miller 1964). It is thus evident that chromosomal investigations now participate, to a significant extent, in differential diagnosis in a variety of sexual abnormalities in man.

In the author's laboratory, chromosomal screening has been carried out since 1959 on patients with congenital and clinical disorders, as well as on those with sexual anomalies, in collaboration with his co-workers and clinicians. Chromosomal features from 300 subjects with congenital disorders and sexual abnormalities were presented recently in a summarized paper (Makino 1964), with some appropriate comment on the reported subjects. In the present report data are presented on both clinical and cytological findings in 150 cases with sexually abnormal development who had been screened in the author's laboratory for chromosomal diagnosis during a period from September, 1959, to July, 1963. The chromosomes of those subjects were mostly already described in some 20 different papers by the author in collaboration with his colleagues and clinicians working in various medical fields. It would not be uncalled for to collect these data, together with some that is unpublished, in one paper, for the sake of convenience to better understand chromosome abnomalities and their correlation with phenotypical effects. Such a summarized report would serve to a certain extent as useful criteria for the significance of the chromosome condition in the etiology of congenital syndromes and clinical defects. It should be mentioned that data presented here are partly inadequate due either to the lack of complete clinical examinations or to limited cytological studies.

Among the 150 cases dealt with in this paper, 44 were found to be chromosomally abnormal. They represent as follows : 29 cases of Klinefelter's syndrome with a regular XXY sex-chromosome complement, one case each of Klinefelter's syndrome with an XXXXY, an XXXY, an XXYY and an XX /XXY sex-chromosome complement, 3 cases of Turner's syndrome with an 
$\mathrm{XO}$ sex-chromosome complement, 1 case of phenotypic female with an XXX sex-chromosome complement, 3 cases of phenotypic males with an $\mathrm{XO} / \mathrm{XY}$ sex-chromosome mosaicism, 3 cases of phenotypic males with a long $Y$ chromosome, and 1 case of phenotypic male with an enlarged short arm on one of no. 15 chromosomes. The remaining 106 cases were found to possess apparently normal chromosome constitutions; they had either normal male or female karyotypes. Data at hand, however, indicated that the unusually long $\mathrm{Y}$ chromosome and the aberrant no. 15 chromosome were not essentially associated with a specific phenotypical anomaly, since similar chromosomal abnormalities occurred in some phenotypically normal and healthy individuals. It was further found that such anomalies were hereditary characters.

It has been repeatedly noted by the author that identifiable chromosomal anomalies or changes alone may not always be associated with the etiology of human clinical disorders, and that a morphologically normal chromosomal complement does not always imply a normal genetic pattern. This is due to the fact that it is more difficult to demonstrate minute loss or rearrangement of chromosomes than it is to detect through current cytological techniques. Attention should be called to the fact that certain abnormal sexual characters may result from some environmental factor (or factors) which causes endocrine unbalance and affects the course of sexual differentiation and development in a certain critical stage after the genetic sex had been normally determined (Makino 1964).

In order to establish the relationship between chromosomal conditions and disease states, there are many problems remaining for future research, and therefore it is still desirable to collect reliable data on a large scale and as broad as possible through survey work.

\section{Acknowledgments}

The author wishes to express his great indebtedness to colleagues who dealt with chromosome analyses in the author's laboratory in association with the author or under his guidance, as well as to clinicians and physicians who associated as joint workers with the author, or who assisted him in collecting materials, and for clinical diagnosis of the subjects described in this paper: Drs. M. J. Dave, J. Fụjieda, S. Fujimoto, M. Hikita, T. Ikeuchi, S. Itoga, T. Kajii, H. Kato, K. Kawakura, Y. Kikuchi, H. Kumakiri, T. Makita, E. Matsunaga, K. Minamiura, M. Mitani, M. Miyamoto, M. Mizutani, S. Morita, J. Muramoto, M. Nagahata, Y. Nakagome, G. Ogawa, T. Omura, M. Rokujyo, M. Sakakura, Masao S. Sasaki, Motomasa Sasaki, S. Shimamura, T. Sofuni, N. Suwa, S. Tabata, N. Takagi, S. Takai, T. Takatsu, M. Tamafuji, I. Tsuji, A. Tsuruta, K. Yamada, N. Yamada, M. Yoshida and $\mathrm{K}$. Yoshikawa. The present study was made possible through their enthusiastic work or assistance. Particularly, the aurhor's cordial thanks are extended to Drs. Motomichi Sasaki and Nobuo Takagi for their expert assistance in preparing essential subjects constituting this article. Further, the author is obliged to Drs. Kazuhide Kuroda and Seiichiro Fujimoto, School of Medicine, Hokkaido University, for helpful advice on clinical conditions. 


\section{References}

Armstrong, C. N. 1964. Intersexuality in man. Intersexuality in vertebrates including man. Academic Press, Lond. \& N. Y. pp. 349-393.

Bierich, J. R. 1963. The adrenogenital syndrome. Intersexuality. Academic Press, pp. 345386.

Court Brown, Harnden, W. M., Jacobs, D. G., Maclean, P. A., N. and Mantele, D. G. 1964. Abnormalities of the sex chromosome complement in man. Privy Council Medical Research Council Special Report Series No. 305.

Dave, M. J. 1966. Cytogenetic studies in 14 sexually abnormal patients. Cytologia 31: 11-18.

Ford, C. E., Jones, K. W., Polani, P. E., De Almedia, J. C. and Briggs, J. H. 1959. A sexchromosome anomaly in a case of gonadal dysgenesis (Turner's syndrome). Lancet i: 711-713.

Hamerton, J. L. 1961. Sex chromatin and human chromosomes. Intern. Rev. Cytol. 12: 1-68.

Harnden, D. G. and Jacobs, P. A. 1961. Cytogenetics of abnormal sexual development in man. Brit. Med. Bull. 17: 206-212.

Hauser, G. A. 1963. Testicular feminization. Intersexuality. Academic Press, pp. 255-276. Hungerford, D. A., Donnelly, A. J. and Nowell, P. C. 1964. The chromosome constitution of a human phenotypic intersex: reconfirmation of a 46-chromosome, XX, apparently non-mosaic "true hermaphrodite". Hereditas 52: 379-386.

Jacobs, P. J. and Strong, J. A. 1959. A case of human intersexuality having a possible XXY sex-determining mechanism. Nature 183: 302-303.

- Harnden, D. G., Buckton, K. E., Court Brown, W. M., King, M. J., Bride, J. A., MacGregor, T. N. and Maclean, N. 1961. Cytogenetic studies in primary amenorrhoea. Lancet i: $1183-1188$.

Kato, H., Omura, T., Tabata, S. and Makino, S. 1965. A preliminary note on an aberrant autosome (group 13-15) in a male child and in his father. Proc. Japan Acad. 41: $477-481$.

Källén, B. and Levan, A. 1962. Abnormal length of chromosome 21 and 22 in four patients with Marfan's syndrome. Cytogenetics 1: 5-19.

Lejeune, J. M., Gautier M. et Turpin, R. 1959. Etude des chromosomes somatiques de neuf enfants mongoliens. C. R. Acad. Sci. (Paris) 248: 1721-1722.

Makino, S. 1961. A chromosome study of man; its history and the present status (in Jaranese). Nippon Rinsho 19: 2186-2204.

- 1962. Chromosome studies in patients with congenital diseases and sex abnormalities in Japanese. Chromosome Information Service 3: 20-21. (Abstract).

- 1964. Chromosome studies in normal human subjects and in 300 cases of congenital disorders. Parts I-III. Cytologia 29: 13-31, 115-150, 233-262.

-, Tonomura, A., Takai, S. and Matsunaga, E. 1960. Chromosome studies in four human phenotypic intersexes (preliminary report). Proc. Japan Acad. 36: 664-669.

- and Kikuchi, Y. 1962. Chromosome studies in several sexually abnormal human subjects. Proc. Japan. Acad. 38: 124-128.

-, Sasaki, M. S. and Kajii, T. (Makino et al.) 1962a. Chromosome studies in eight cases of human congenital diseases. Proc. Japan Acad. 38: 166-170.

-, Tonomura, A., Mizutani, M., Sakakura, H., Kumakiri, S. Itogo, S. and Yamafuji, M. (Makino et al.) 1962b. Chromosome studies in five sexually abnormal patients. Proc. Japan Acad. 31: 31-36.

-, Yamada, K. and Kajii, T. (Makino et al.) 1962c. Chromosome studies in seventeen cases of congenital diseases. Proc. Japan Acad. 38: 758-762.

-, Yoshida, M., and Mitani, M. (Makino et al.) 1962d. Chromosome studies in ten cases of human congenital diseases. Proc. Japan Acad. 38: 356-368.

- and Sofuni, T. 1963. Chromosomes of eleven sexually abnormal patients. J. Fac. Sci. Hokkaido Univ. Ser. VI, Zool. 15: 248-253. 
-, Sasaki, M., Sasaki, M. S., Kikuchi, Y., Mizutani, M., Nagahata, M., Nakagome, Y. and Yoshikawa, K. (Makino et al.) 1963a. Chromosome studies in 37 cases of human congenital diseases. Proc. Zool. Soc. Calcutta, India 16: 1-22.

-, Sofuni, T. and Yamada, K. (Makino et al.) 1963b. Chrohosome studies in twelve sexually abnormal patients. Proc. Japan Acad. 39: 241-246.

-, Sasaki, M.S., Yamada, K. and Kajii, T. (Makino et al.) 1963c. A long Y chromosome in man. Chromosoma (Berl.) 14: 154-161.

- and Muramoto, J. 1964. Some observations on the variability of human Y chromosome. Proc. Japan Acad. 40: 757-761.

-, Makita, T., Muramoto, J., Kajii, T. and Mitani, M. (Makino et al.) 1964a. Chromosome studies in fourteen cases of human congenital disorders. Proc. Japan Acad. 40: 291-295.

-, Takagi, N. and Hikita, M. (Makino et al.) 1964b. Two phenotypic males with XXXXY and XXXY sex chromosomes. Proc. Japan Acad. 40: 427-431.

-, -, Makita, T. and Hikita, M. (Makino et al.) 1964c. A chromosome study of ten sexually abnormal patients. Proc. Japan Acad. 40: 566-571.

-, -, Miyamoto, N., Sasaki, M. S., Morita, S. and Sasaki, M. (Makino et al.) 1964d. Chromosomes of sixteen fatients with congenital diseases. Proc. Japan Acad. 40: 134139.

- and Takagi, N. 1966. Some morphological aspects of the abnormal human Y chromosome. Cytologia 30: 274-292

-, Muramoto, J. and Tabata, S. 1966. A survey of a familial transmission of an anomalous autosome in group 13-15. Chromosoma (Berl.) 18: 371-379.

Miller, O. J. 1964. The sex chromosome anomalies. Am. J. Obst. \& Gynec. 90: 1078-1139.

Robinson, A. Priest, R. E. and Bigler, P.C. 1964. Male pseudohermaphrodite with XY/XO mosaicism and bilateral gonadoblastomas. Lancet i: 111-112.

Sasaki, M. and Makino, S. 1960. The chromosomal constitution of a human hermaphrodite. Texas Rep. Biol. Med. 18: 493-500.

Takagi, N., Kato, H., Takai, S. and Hikita, M. 1966. A chromosome survey in 40 sexually abnormal patients. Jap. Jour. Genet. 41: 1-7.

-, Makino, S., Takai, S. and Hikita, M. 1965. A phenotypical XXYY human male with notes on two regular Klinefelter cases. Proc. Japan Acad. 41: 175-180.

Tjio, J. H. and Levan, A. 1956. The chromosome number of man. Hereditas 42: 1-6.

The descriptions regarding clinical and cytogenetical features of the cases dealt with in this paper will appear in more detail in the next issue in the form of an appendix [Makino, S. Chromosome studies in 150 sexually abnormal patients (A summarized report), Part II. Cytologia Vol. 31, No. 4, 1966]. 